\title{
A Practical Approach to SIP, QoS and AAA Integration
}

\author{
Michael Stier, Emanuel Eick, and Eckhart Koerner \\ University of Applied Sciences Mannheim, \\ Institute for Software Engineering and Computer Networks, \\ Faculty of Information Technology, \\ Windeckstr. 110, 68163 Mannheim, Germany \\ \{m.stier, e.eick, e.koerner\}@hs-mannheim.de
}

\begin{abstract}
In this paper, we present an overall architecture to integrate SIPbased session management with scalable QoS features and AAA functionality. The QoS features include a bandwidth reservation in the access network based on the Next Steps in Signaling (NSIS) architecture. Furthermore, we employ the DiffServ compliant Priority Promotion Scheme (PPS) which is a packet probing scheme to verify end-to-end premium bandwidth availability. To avoid misuse of the QoS features AAA functionality is added that binds the QoS usage with the specific SIP sessions. The AAA functionality is achieved by applying a combination of the widespread RADIUS protocol with the Common Open Policy Service (COPS). As a proof of concept our architecture has been implemented and performance tested. The strength of our approach is that it keeps away complexity from the carrier networks while providing QoS with access control in a scalable fashion.
\end{abstract}

Keywords: SIP, QoS, AAA, real-time interactive services, bandwidth probing.

\section{Introduction}

Usage of real-time voice and video services over fixed and wireless access networks to the Internet is steadily increasing while Quality of Service (QoS) features for such services are still absent. For instance, on a DSL link a Voice over IP call may still be easily disturbed by competing data traf120fic. The rollout of existing QoS architectures is especially not progressing into the access networks as solutions for admission control to QoS features are still too complex. In this paper, we present a novel architecture that combines advanced and yet simple QoS techniques with proven AAA technologies. This combination is then integrated with SIP-based session management.

Our approach to QoS is twofold. In the access networks, we suggest bandwidth reservations for real-time voice and video where the QoS requirements are signalled through the new Next Steps in Signaling protocols. As the associated streams should be transported as premium traffic in the carrier networks we additionally propose an end system-based bandwidth probing scheme that verifies the availability of end-toend premium bandwidth. As the best candidate for this purpose we have identified the Priority Promotion Scheme, short PPS, which is DiffServ compliant.

Radius is the well-established and most-widespread protocol to provide AAA functionality for Internet access. Consequently, we take it as the basis to control access to QoS features. It can be supported by COPS to enforce a binding between the SIP-based session management and the QoS features. 
The next chapter introduces the fundamentals of SIP, PPS, NSIS and the relevant AAA architectures and protocols. From there, we derive our architecture which is presented in chapter 3. In chapter 4, we demonstrate how our architecture was implemented with open source software. The behaviour of the resulting environment is evaluated in chapter 5 . We eventually draw our conclusions in chapter 6 .

\section{Related Work}

In this chapter, we introduce all the ingredients needed to design our architecture, namely SIP, PPS, NSIS and AAA.

\subsection{Session Management with SIP}

The Session Initiation Protocol (SIP) [1] is a multimedia session management protocol. In conjunction with the Session Description Protocol (SDP) [2] it is the signalling protocol of choice for real-time interactive applications, including in particular Voice over IP (VoIP).

\subsection{Priority Promotion Scheme}

With the Priority Promotion Scheme (PPS) [3, 4] session-based on-demand Quality of Service (QoS) can be provided end-to-end in a scalable fashion. PPS uses probing packets that are sent by end systems before the actual data traffic in order to estimate the present state of a network path. The receiver of probing packets can estimate the quality of a path on the basis of packet loss or jitter. PPS is also DiffServ [5]compliant. In a Diffserv network, a classification of traffic into differently prioritized classes is made. By means of traffic conditioning all incoming packets on a router are treated according to their traffic class. For policing, probing packets are distinguished from other packets by a different Diffserv Code Point (DSCP). For instance, IP premium traffic may be marked with the DSCP value of 46 for Expedited Forwarding Per-Hop Behaviour (EF PHB) and the associated probing packets may be marked with a DSCP of 47 . On the egress interface of a router a maximum rate of

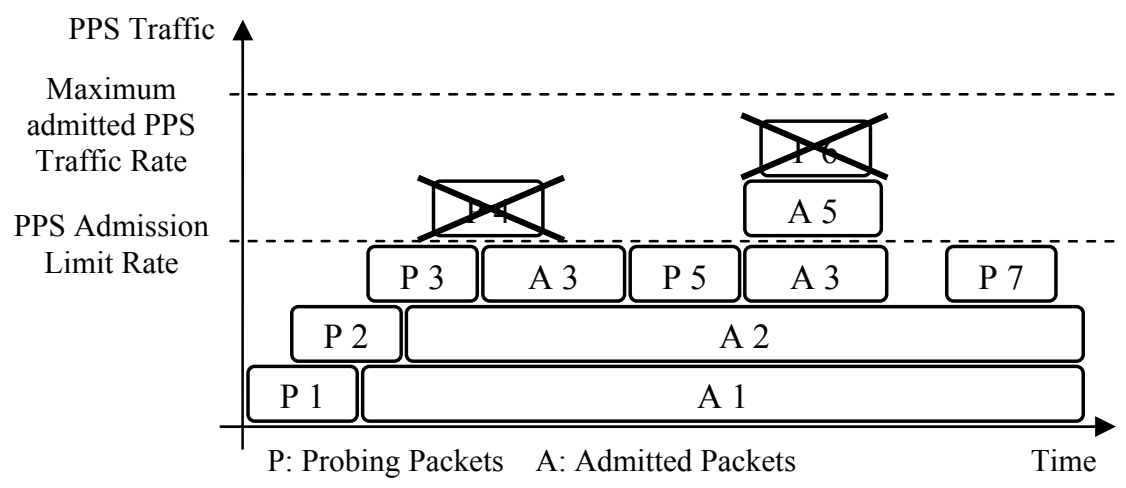

Fig. 1. Principle of PPS 
PPS traffic,i.e. sum of probing and IP premium traffic, is configured (see fig. 1). Below this maximum a threshold for PPS admission control is defined. Whenever this threshold is exceeded, probing packets are dropped while IP premium traffic is still forwarded up to the configured PPS maximum rate. This behaviour guarantees bandwidth for all admitted flows while flows experiencing packet loss or considerable jitter in the probing phase are rejected.

\subsection{QoS Signalling with NSIS}

The IETF working group Next Steps in Signalling (NSIS) is working on a new flow signalling framework [6, 7]. NSIS is based on a two layer paradigm (see fig. 2). The lower layer is the NSIS Transport Layer Protocol (NTLP) [8] which resides on top of standard transport protocols. NTLP is responsible for delivering signalling messages from a flow source to a flow destination. The higher layer is the application-specific signalling layer with various NSIS Signalling Layer Protocols (NSLPs). For instance, NSLPs exist for NAT/firewall configuration and in particular for resource reservation signalling - the QoS NSLP [9].

At least the messaging layer must be provided for NSIS signalling on all involved network entities. The signalling sequence for QoS NSLP is depicted in fig. 3. The initiating entity is called QoS NSIS initiator (QNI). A QNI request passes one or more intermediate entities (QNE) along the NSIS path and finally terminates at the QoS NSIS responder (QNR). The request will be processed by the responder's client layer and the response travels along the reverse path to the QNI.

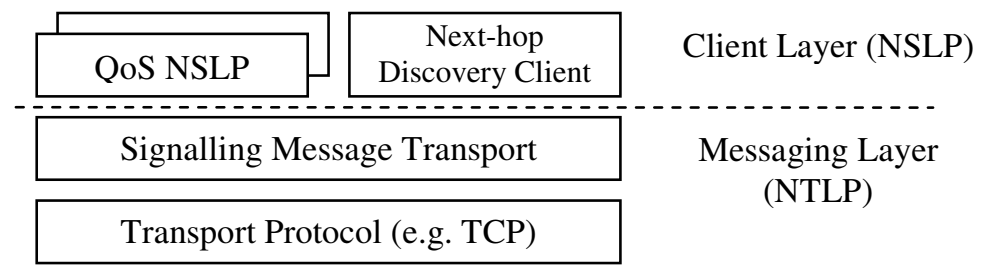

Fig. 2. NSIS architecture

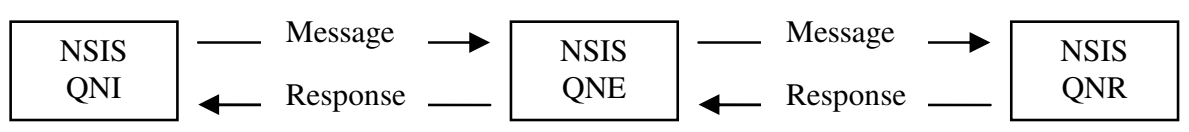

Fig. 3. NSIS entities for QoS signalling

\subsection{Authentication, Authorization and Accounting (AAA)}

A major interest of a service provider is that specific service features like QoS can only be used by subscribed customers. Therefore, it is important to authenticate users and authorize the usage of value added services. AAA servers are a solution for this problem. For the examination of user credentials the Remote Authentication Dial In User Service (RADIUS) [10] is the most widely deployed solution. RADIUS is 
characterized by its simplicity and its versatile possibilities. Although with Diameter [11] a successor is already standardized, there is no significant Diameter deployment yet.

It is very difficult to manage all entities in an ISP's infrastructure consistently, especially when these elements cooperate to provide a common service feature like QoS. Policy servers can be used to manage an administrative domain from a centralized point. To support such a policy concept the Common Open Policy Service (COPS) [12] was developed. COPS is a classical query/response protocol for exchanging policy elements between a policy server and its clients. COPS needs a socalled Policy Enforcement Point (PEP) which interprets the policy elements, received from the policy server's Policy Decision Point (PDP) [13]. The PEP has to obey the policy server's decision when performing the requested service.

\section{Architecture}

In the previous chapter, we have introduced several components that can be combined into a system for real-time interactive services. In our approach, the goal was to design a system which meets the requirements of an ISP. At the same time, the provisioning of QoS as an added value and its protection against misuse were of primordial importance.

The QoS part of our system takes into account the traffic situation in the Internet. The bandwidth in the access networks (e.g. mobile networks, xDSL) is scarce while the core networks are highly overprovisioned. It is expected that this situation will prevail for many years to come. With respect to QoS for real-time interactive services, this situation is problematic. For instance, on an ADSL access line a VoIP call can easily be disturbed by simultaneous data traffic from $\mathrm{p} 2 \mathrm{p}$ applications or web downloads. The core network normally has sufficient bandwidth to transport both streams, but usually does not provide expedited forwarding for real-time interactive traffic.

Therefore, our architecture supports an explicit bi-directional reservation of bandwidth for real-time interactive services from the end systems to the network access router of the ISP. For that purpose, we adopt the QoS NSLP as described in section 2.3. Furthermore, to enable expedited forwarding of packets in DiffServ enabled core networks we employ the PPS scheme as described in section 2.2 after a successful reservation of access bandwidth has been made (see fig. 4).

As the QoS signalling is initiated by end users, there are potential security vulnerabilities. A malicious user could, for example, forge QoS signalling messages in order to gain access to prioritized service classes. The security requirements can be met by the assistance of the AAA components introduced in section 2.4. Concretely, our design choice was inspired by the token based models of the IETF's "Framework for Session Set-up with Media Authorization" [14]. In the so-called coupled model our most important requirement is addressed, namely the explicit binding of the QoS feature to a specific service. A token is issued by the AAA components to authorize usage of a service feature, in our case QoS, by the end user application. This token is fed back by the end user application into the QoS NSLP requests. Afterwards it is extracted by the access router and passed back to the policy server, as shown in fig. 4 . The policy server acting as a Policy Decision Point (PDP) finally verifies the token's validity. The message loop which arises from this sequence gave it the name coupled 


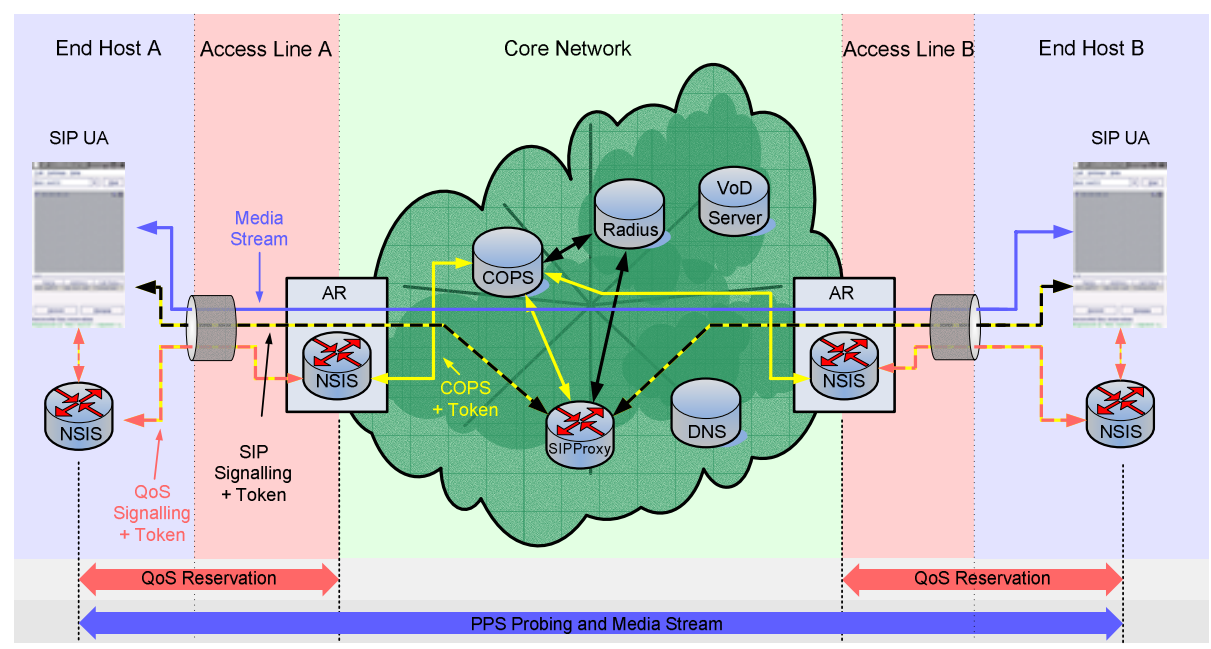

Fig. 4. Architecture integrating SIP signalling, PPS and QoS reservation with AAA

model. The model includes a Session Management Server (SMS) as an entity that provides session management services and functions as a Policy Enforcement Point (PEP). In our architecture, the SMS is a SIP Proxy.

We have integrated the coupled model with the SIP architecture according to the "Private Session Initiation Protocol (SIP) Extensions for Media Authorization" [15]. The Token is transported in an additional P-Media-Authorization SIP header field. For the token structure we adopted the IETF's "Session Authorization Policy Element" specification [16]. The token is packaged as a session identifier into the recommended policy element structure. It associates the QoS reservation with the SIP service preventing misuse of an established QoS reservation for other services.

The SIP call setup also needs to be integrated with the PPS procedure. This has been done in accordance with the specification for the "Integration of Resource Management and Session Initiation Protocol (SIP)" [17]. According to the scientific research on probe-based admission control the recommended duration of the probing phase is about one second [18]. The probing packets are sent bi-directionally between SIP User Agents (UAs). Frequency and size of the probing packets depend on the negotiated codecs. The information about available codecs is exchanged in the media description header field of the Session Description Protocol (SDP) [2] as part of SIP session setup messages prior to the probing. After the probing has finished each client analyses the received probing packets. If there are lost or truncated packets one can draw the conclusion that the network cannot provide the required end-to-end bandwidth. Otherwise, if the packet loss is zero the call setup can continue.

The SIP UA of the caller signals a busy line (all trunks busy) if the NSIS bandwidth reservation fails or the PPS function detects an insufficient end-to-end network path. The user is hence saved from a call with an inadequate quality. On the other side, if the NSIS reservation was successful and the PPS function confirms the availability of premium resources the call will be established. The negotiated media streams are established and marked with the DSCP value of 46 for expedited forwarding. 
The details of the interaction between all components are illustrated by the message sequence chart in fig. 5 .

The most relevant messages of the flow are:

1. The SIP User Agent Client (SIP-UAC) wants to gain access to a distinct service. It initially sends an INVITE message to the SIP proxy which challenges the client to authenticate itself with a digest authentication message [19]. The session description additionally contains the media type that is supposed to receive the QoS treatment.

2. The SIP proxy authenticates the client for the SIP service and forwards the digest authentication message to the policy server.

3. The policy server creates a request message including the digest authentication message and forwards it to the RADIUS Server.

4. The RADIUS Server validates the digest authentication message and creates a response containing the QoS status. This message is sent back to the policy server.

5. The policy server creates a token wrapping the QoS status. This token is returned to the SIP proxy.

6. Steps 4 and 5 are repeated to generate a second token for this session. After this step the authentication and authorization phase is finished.

7. The SIP proxy forwards the first token to the callee (SIP-UAS).

8. The second token is forwarded to the caller (SIP-UAC).

9. The SIP user agent is requesting resources. It creates a RESERVE message containing the token that is transferred through the UAC-QNI to the access router (AR-1 QNR) performing the QoS reservation.

10. The access router extracts the token and forwards it in a POLICY REQUEST message to the policy server.

11. The policy server searches its internal database using the token as a key. If a matching entry can be found, the stored credentials are checked and a decision is made whether the token is still valid. At this stage the loop of the token is closed.

12. If the token was successfully validated, the QoS information is packed into a positive decision message. It is sent to the entity requesting the QoS status. The policy server removes the database entry of this token so that a re-use of this token becomes impossible.

13. The AR interprets the decision message and performs the QoS reservation.

14. The decision whether a reservation was made is sent back to the requesting entity.

15. The steps 9 to 14 are repeated simultaneously on the side of the callee (SIP-UAS) with its token and access router (AR-2 QNR).

16. After the QoS state is established the probing phase begins.

17. The probing phase ends. In case of an affirmative response the user agents are synchronizing themselves with a SIP UPDATE message. The RINGING message follows. In case an insufficient network quality is detected the session will be cancelled.

18. The subsequent messages are the same as in a usual SIP call flow.

This message flow ensures that the QoS support is only granted to a valid subscriber of the SIP service. By today's standards it is impossible for an attacker to manipulate or reuse the tokens without the detection of the fraud by the policy server. 


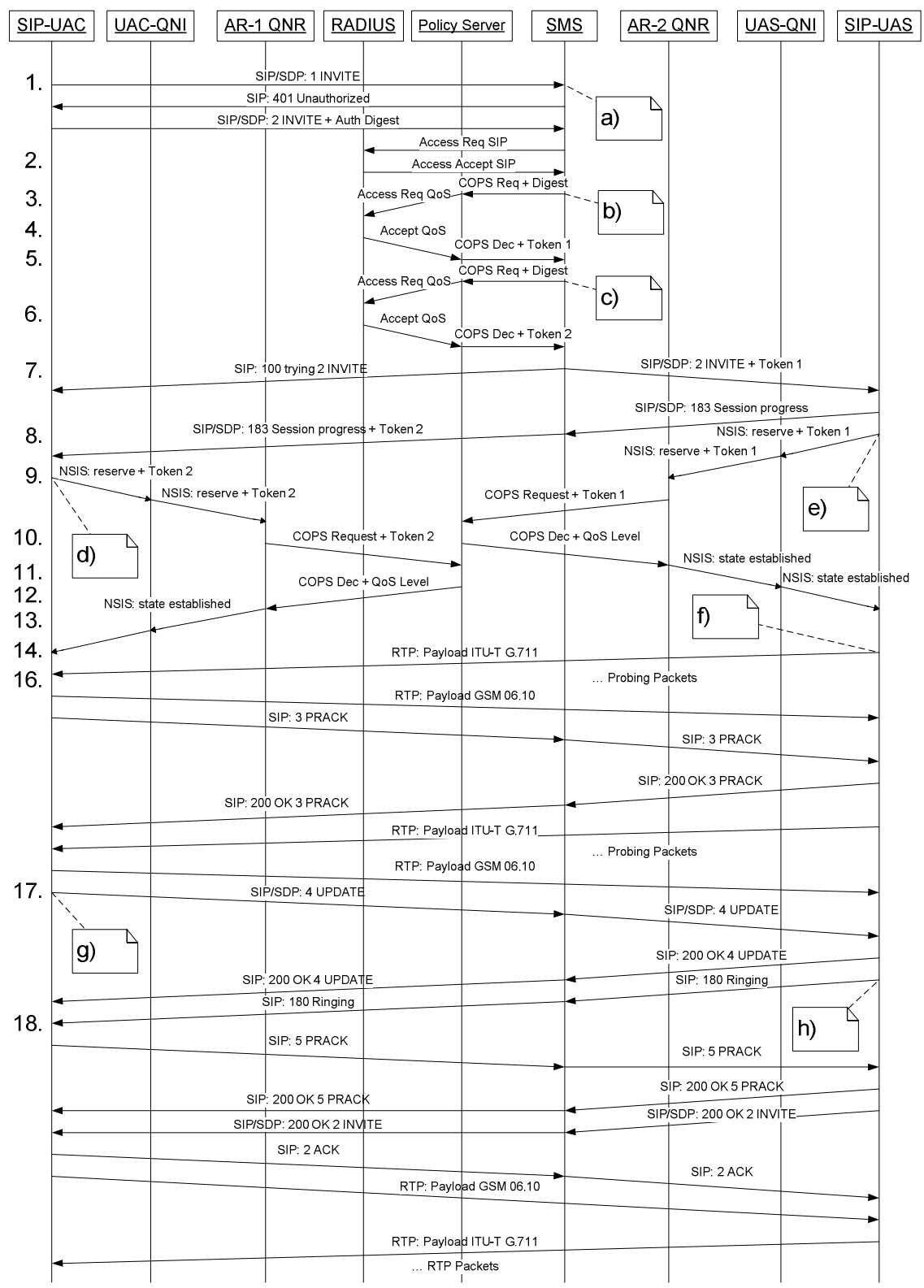

Fig. 5. SIP Call flow with PPS, QoS Signalling and AAA Coupled Model

Not shown in the figure are the REFRESH messages during the established call and the termination of the call. Due to the soft-state nature of the NSIS design each established QoS reservation has to be refreshed at an average of every 60 seconds. Otherwise, the NSIS stack has to time out the QoS reservation. If a user hangs up, the SIP session will be finished and the QoS reservation will be torn down. 


\section{Implementation}

The presented architecture was implemented for validation and demonstration. We have realized an entire VoIP system consisting of SIP UAs, a SIP proxy, a RADIUS server, a policy server and the NSIS stack on each box. The components were distributed over 4 boxes. Table 1 shows the configuration of each box.

Table 1. Configuration of the VoIP system

\begin{tabular}{|c|c|c|c|c|}
\hline & Box 1 & Box 2 & Box 3 & Box 4 \\
\hline Tasks & $\begin{array}{l}\text { SIP-UA 1 } \\
\text { QNI 1 }\end{array}$ & $\begin{array}{l}\text { SIP-UA } 2 \\
\text { QNI } 2\end{array}$ & $\begin{array}{l}\text { SIP Proxy, AR 1, } \\
\text { QNR 1, RADIUS, } \\
\text { policy server, DNS }\end{array}$ & $\begin{array}{l}\text { AR 2, } \\
\text { QNR 2 }\end{array}$ \\
\hline $\mathrm{OS}$ & Linux 2.6.10 & Linux 2.6.12 & Linux 2.4 .9 & Linux 2.6.10 \\
\hline Processor & $\begin{array}{c}\text { Mobile P3 } \\
1 \mathrm{GHz}, 256 \mathrm{MB}\end{array}$ & $\begin{array}{c}\mathrm{M} 715 \\
1,5 \mathrm{GHz}, 512 \mathrm{MB}\end{array}$ & $\begin{array}{c}\text { Mobile P3 } \\
1 \mathrm{GHz}, 384 \mathrm{MB}\end{array}$ & $\begin{array}{c}\text { PII } 800 \mathrm{MHz} \\
256 \mathrm{MB}\end{array}$ \\
\hline
\end{tabular}

Some of the components and their implementation are described subsequently.

\section{Sip User Agent}

The starting point for our extensions was the open source "SIP Communicator" user agent from the java.net project [20] which is developed at the Université Louis Pasteur in Strasbourg. First of all, we integrated the PPS procedure into this software UA. Every UA also needs one NSIS entity as initiator of the QoS signalling path. Since there is no NSIS implementation in the product stage we reused an implementation of the NSIS-like Cross Application Signalling Protocol (CASP) from University of Goettingen [21]. CASP is a predecessor of NSIS that is compliant to the requirements specified by the NSIS working group. The replacement of the CASP components with future QoS NSLP/GIST modules that are currently developed at the University of Goettingen should be straightforward. The CASP modules have been written in C. They are integrated into the Java client via a Java/C Wrapper [22]. The AAA functionality of the SIP communicator is doing the forwarding of the received token to the QoS NSIS initiator.

\section{Network Access Router}

One NSIS entity for path termination is deployed on each network access router. At the signalling layer we are using the QoS Client specified in [23] which has been implemented by the University of Goettingen [24]. Associated to this module is a COPS client, which initiates the verification of the token by the policy server. The token is extracted from the POLICY_DATA object in the CASP RESERVE message. The PPS probing procedure requires a suitable policer. This policer which is currently developed at the University of Goettingen will be integrated into the environment in the near future. It is based on the hierarchical token bucket packet scheduler available in most LINUX kernels [25].

\section{SIP Proxy and AAA}

The widely used SIP Express Router (SER) from Iptel has been installed as a SIP proxy running in version 0.9.3 on LINUX [26]. We have additionally installed the SER 
RADIUS module including the RADIUS client which provides the interface for the interaction with the RADIUS server [27]. Furthermore, we developed a COPS client module for the SER that provides the interaction with the policy server for the authorization of QoS usage. The DNS holds the required SRV records for the call domain.

\section{Evaluation}

Based on the demonstrator the performance of the system has been studied. First of all, we discuss the timing behavior of the call flow from section 3. Figure 6 is showing the packet numbers over time beginning with the initial SIP INVITE message from the UAC right up to the RINGING message originating from the UAS.

Relevant points in time are indicated by the markers a) to $h$ ) referring to the call flow in figure 5. Table 2 provides details for each marker.

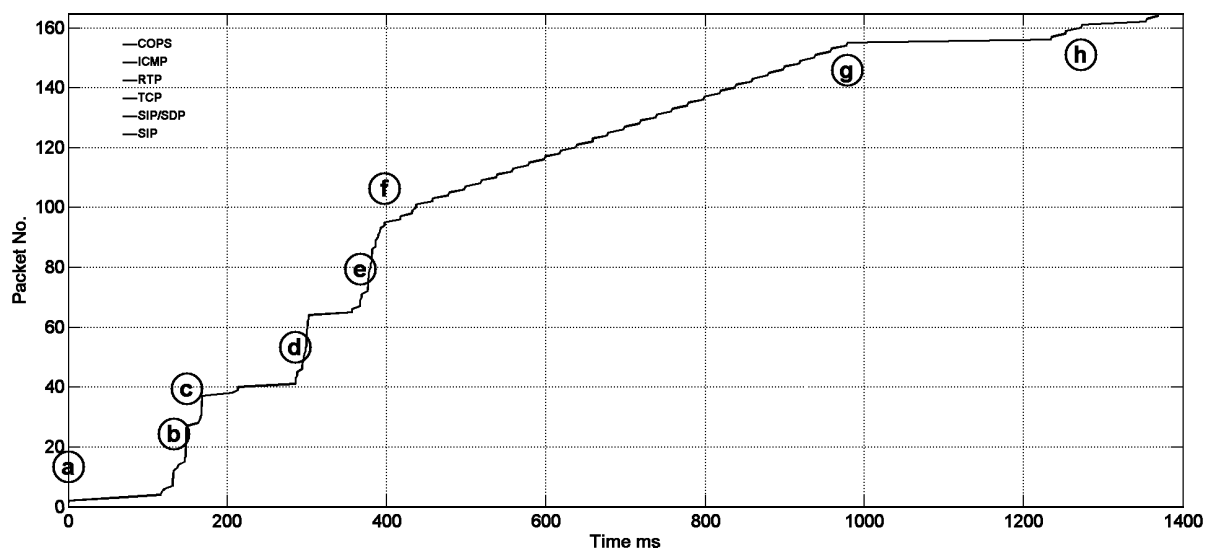

Fig. 6. Time table of SIP call flow from INVITE until RINGING message

Table 2. Time table of markers in the SIP call flow from INVITE until RINGING message

\begin{tabular}{|c|c|c|l|}
\hline Marker & Packet No. & Elapsed Time & \multicolumn{1}{|c|}{ Description } \\
\hline a) & 1 & $0 \mathrm{~ms}$ & INVITE \\
\hline b) & 12 & $132 \mathrm{~ms}$ & COPS request for token 1 \\
\hline c) & 27 & $149 \mathrm{~ms}$ & COPS request for token 2 \\
\hline d) & 41 & $286 \mathrm{~ms}$ & UAC QoS reservation \\
\hline e) & 67 & $367 \mathrm{~ms}$ & UAS QoS reservation \\
\hline f) & 94 & $397 \mathrm{~ms}$ & First PPS probing packet \\
\hline g) & 156 & $981 \mathrm{~ms}$ & Last PPS probing packet \\
\hline h) & 160 & $1272 \mathrm{~ms}$ & RINGING \\
\hline
\end{tabular}


Nearly $60 \%$ of the packets are sent in the first $400 \mathrm{~ms}$. One reason for this large number of packets is that the NSIS QoS signalling uses non-persistent TCP transport.The longest period of time $(600 \mathrm{~ms})$ is needed for the probing phase marked from f)until g). It can be seen that the Java SIP UA responds relatively slow. For example, the UA needs $250 \mathrm{~ms}$ processing time to reply with an UPDATE message after the probing phase. This test was done on box 1 to box 4 as listed in table 1 . The overall time of about $1300 \mathrm{~ms}$ for call setup is fully acceptable.

Special attention should be paid to the server components. First, we look at the RADIUS server. We wanted to know how long it takes to do a digest authentication in contrast to a simple username/password authentication. Therefore, we have done performance tests to identify the processing time for one digest authentication in a test series comprising from 10 to 100.000 requests. With rising number of requests the processing time converges in case of digest authentication to $1677 \mu$ s and in case of username/password authentication to $1624 \mu \mathrm{s}$. Thus, one can conclude that digest authentication requires only about 3 percent more processing time. This test was done on a Pentium M715 box with 512 MB Ram and Linux OS 2.6.12.

In another analysis we studied the required extra time for the AAA functionality, especially for the token-based authorization. We measured the SER processing time, i.e. the overall time needed from creating a COPS request in the PEP until the COPS decision is interpreted. In detail, the SER processing time includes the RADIUS time, the time the policy server needs to send a RADIUS access request and receive the corresponding answer and the COPS processing time, which is the time span for creating a valid COPS decision message containing the token. The plot in fig. 7 is showing the timing for each benchmark cycle on a P4 3.2GHz Linux OS 2.6.11 machine.

The mean time of $11 \mathrm{~ms}$ to perform the authorization is satisfactory. It is a fair tradeoff considering the security gained against misuse of the QoS feature. The time for the verification of the token by the policy server can be neglected, as this task takes less than $4 \mathrm{~ms}$.

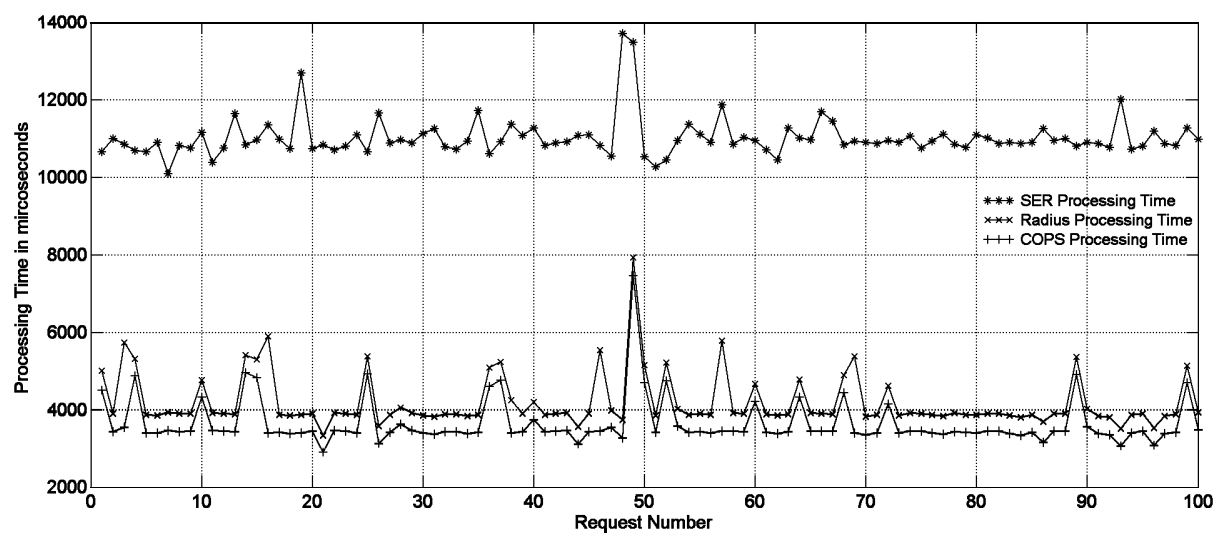

Fig. 7. Processing time for user authorization and for issuing the token 


\section{Conclusions}

We have presented a comprehensive architecture to integrate SIP, QoS and AAA. Our architecture provides a QoS reservation on the access link that the SIP UAs initiate with NSIS. The state of the end-to-end network path is probed with PPS. PPS adheres to DiffServ. AAA functions prevent misuse of the QoS support. Only users possessing a validated token can benefit from QoS. A prototype of this architecture was implemented to verify the concepts.

PPS has been studied scientifically before. We have designed the first practical application of PPS in the context of multimedia session management. As an endsystem based admission control scheme it perfectly fits the Internet philosophy that puts the complexity into the end systems. As such, PPS is also considered in the Multiparty Multimedia Session Control Working Group (mmusic) [28] where endsystem based admission control is on the charter.

We have used a pairing of COPS and Radius to support AAA. When Diameter becomes more widespread in the future our architecture can easily be mapped onto a Diameter QoS application.

Finally, the presented architecture is applicable to session management in general, including in particular the Real Time Streaming Protocol (RTSP) [29]. In the latter case, one will only have to deal with unidirectional streams from the streaming server to the media players which will make NSIS signalling and PPS probing even less costly.

\section{Acknowledgements}

The work presented in this paper has been performed as part of the project GOSSIP which is funded by the German Federal Ministry of Education and Research (BMBF) under grant number $1719 \mathrm{X} 04$. We would especially like to acknowledge many fruitful discussions with our GOSSIP project partners at T-Systems, in particular Mr. Rüdiger Geib who drew our attention to PPS and NSIS. We also thank Xiaoming Fu and Ingo Juchem from University of Goettingen for the support related to the NSIS stack.

\section{References}

1. Rosenberg, J. et al.: SIP: Session Initiation Protocol. IETF RFC 3261, June 2002

2. Handley, M., Jacobson, V.: SDP: Session Description Protocol. IETF RFC 2327, April 1998

3. Mori, S., Kawarasaki, Y., Kataoka, H. and Morita, N.: Priority Promotion Scheme (PPS) An Autonomous and Distributed Admission Control for End-to-end Quality of Service for Interactive Multimedia Services, NTT Technical Review Online, October 2004, http://www.ntt.co.jp/tr/0410/special.html

4. Morita, N., Karlsson, G.: Framework of Priority Promotion Scheme. IETF Internet Draft draft-morita-tsvwg-pps-01, October 2003

5. Blake, S. et al.: An Architecture for Differentiated Services. IETF RFC 2475, Dec. 1998

6. Hancock, R. et al.: Next Steps in Signaling (NSIS): Framework. IETF RFC 4080, June 2005 
7. Fu, X. et al.: NSIS: A New Extensible IP Signaling Protocol Suite. In: IEEE Communications Magazine, Internet Technology Series, page 133-141, IEEE, October 2005

8. Schulzrinne, H. et al.: GIST: General Internet Signaling Transport. IETF Internet Draft draft-ietf-nsis-ntlp-08, work in progress, September 2005

9. Van den Bosch, S., et al.: NSLP for Quality-of-Service signalling. IETF Internet Draft draft-ietf-nsis-qos-nslp-08, work in progress, October 2005

10. Rigney, C. et al.: Remote Authentication Dial In User Service (RADIUS). IETF RFC 2865, June 2000

11. Calhoun, P. et al.: Diameter Base Protocol. IETF RFC 3588, September 2003

12. Durham, D. et al.: The COPS (Common Open Policy Service) Protocol. IETF RFC 2748, January 2000

13. Yavatkar, R. et al.: A Framework for Policy-based Admission Control. IETF RFC 2753, January 2000.

14. Hamer, L-N. et al.: Framework for Session Set-up with Media Authorization. IETF RFC 3521, April 2003

15. Marshall, W. (ed.): Private Session Initiation Protocol (SIP) Extensions for Media Authorization. IETF RFC 3313, January 2003

16. Hamer, L-N. et al.: Session Authorization Policy Element. IETF RFC 3520, April 2003

17. Camarillo, G., Marshall, W., Rosenberg, J.: Integration of Resource Management and Session Initiation Protocol (SIP). IETF RFC 3312, October 2002

18. Más Ivars, I., Karlsson, G.: PBAC: Probe-Based Admission Control. In Proc. of QofIS 2001, vol. 2156 of LNCS, (Coimbra, Portugal), pp. 97-109, Springer, September 2001

19. Franks, J. et al.: HTTP Authentication: Basic and Digest Access Authentication. IETF RFC 2617, June 1999

20. SIP User Agent Sip-Communicator, https://sip-communicator.dev.java.net

21. Schulzrinne, H., Tschofenig, H., Fu, X., McDonald, A.: CASP - Cross-Application Signaling Protocol. IETF Internet Draft draft-schulzrinne-nsis-casp-01.txt, March 2003 http://user.informatik.uni-goettingen.de/ casp/draft-schulzrinne-nsis-casp-01.pdf

22. Simplified Wrapper and Interface Generator (SWIG), http://www.swig.org

23. Schulzrinne, H. et al.: A Quality-of-Service Resource Allocation Client for CASP. IETF Internet Draft draft-schulzrinne-nsis-casp-qos-01.txt, March 2003, http:// user.informatik. uni-goettingen.de/ casp/draft-schulzrinne-nsis-casp-qos-01.pdf

24. Juchem, I.: An Implementation of QoS NSLP. http://user.informatik.uni-goettingen.de/ $\sim$ qos/

25. Severa M.: Hierarchical token bucket (HTB) home. Packet Scheduler, http://www. huisetalage .nl/ sip/index.html

26. Sip Express Router (SER), Free SIP Server, http://www.iptel.org/ser

27. freeRADIUS, Open Source RADIUS Server Project, http://www.freeradius.org

28. Multiparty Multimedia Session Control (mmusic), IETF working group, http://www.ietf. org/ html.charters/mmusic-charter.html

29. Schulzrinne, H. et al.: Real Time Streaming Protocol (RTSP). IETF RFC 2326, April 1998. 